02

\title{
К вопросу о бозе-эйнштейновском конденсате экситонов в кристаллах с дефектами
}

\author{
() Ю.Д. Заворотнев ${ }^{1}$, В.В. Румянцев ${ }^{1}$, С.А. Федоров ${ }^{1}$, Е.Ю. Томашевская ${ }^{2}$ \\ ${ }^{1}$ Донецкий фризико-технический институт им. А.А. Галкина, \\ 83114 Донецк, Украина \\ 2 Донецкий национальный университет экономики и торговли им. Туган-Барановского, \\ 83114 Донецк, Украина \\ e-mail: vladimir.rumyantsev2011@yandex.ru
}

Поступила в редакцию 23.03.2020 г.

В окончательной редакции 23.03.2020 г.

Принята к публикации 20.05.2020 г.

Рассмотрена возможность образования бозе-эйнштейновского конденсата (БЭК) экситонов в модельной неидеальной решетке молекулярного кристалла. Проведен анализ спектра соответствующих экситонных возбуждений. В рамках приближения виртуального кристалла выполнено численное моделирование зависимости химического потенциала от концентрации структурных дефектов и на этой основе изучены особенности появления экситонного конденсата в неидеальной системе. Показано, при каких условиях возможно появление БЭК светлых и темных экситонов.

Ключевые слова: экситон, бозе-эйнштейновский конденсат, химический потенциал, структурные дефекты, приближение виртуального кристалла.

DOI: $10.21883 /$ OS.2020.10.50010.114-20

\section{Введение}

С появлением мощных источников когерентного излучения оказалось возможным создавать в кристаллах плотности экситонов порядка $10^{17}-10^{18} \mathrm{~cm}^{-3}$. В этом случае существенными становятся их коллективные состояния, в частности образование бозе-эйнштейновского конденсата (БЭК). Пионерами в изучении БЭК экситонов является группа С.А. Москаленко [1,2]. Из результатов исследований по обнаружению экситонного конденсата в различных кристаллах, которые ведутся на протяжении 50 лет [3-8], следуют два фундаментальных вывода: 1) БЭК наступает при некоторых критических плотностях экситонов в кристалле; 2) ширина линии люминесценции (одного из способов обнаружения экситонного конденсата) должна быть бесконечно узкой. Важно отметить также то обстоятельство, что экситон является не элементарным, а составным бозоном, и время жизни этих квазичастиц чрезвычайно мало. Последнее в меньшей степени относится к экситонам Ванье-Мотта и в большей - к короткоживущим (светлым) экситонам Френкеля. Дополнительно к названным существуют и другие затруднения в образовании БЭК, часть из которых рассматривается в настоящей работе.

До настоящего времени БЭК экситонов изучался без учета структурных дефектов в кристаллах. Однако сегодня в связи с созданием новых функциональных материалов появляется возможность путем введения в исследуемую систему определенных дефектов добиваться необходимого изменения ее энергетической структуры и, следовательно, значительных изменений оптических свойств, обусловленных перенормировкой энергетического спектра соответствующих квазичастиц. Одной из важнейших характеристик конденсата является химический потенциал, величина которого определяет сдвиг частоты рассеянных волн в нелинейных оптических процессах [9]. Отметим, что химический потенциал БЭК экситонов (в отличие от соответствующей величины для газов [10]) отличен от нуля, поскольку экситоны конденсируются в возбужденное состояние (минимум дисперсионной ветви). Особый интерес представляет изучение БЭК экситонов в неидеальных системах и исследование особенностей зависимости химического потенциала от концентрации структурных дефектов.

В последнее время появились работы, в которых рассматриваются экситоноподобные (типа экситонов Френкеля) электромагнитные возбуждения [11] в неидеальных пористых цепочках и квазидвумерных структурах с порами, а также исследуются экситоны Френкеля и поляритоны в подобных структурах, содержащих квантовые точки [12-14]. В работах [11-14] представлена общая схема нахождения спектра квазичастичных возбуждений неидеальной системы со случайным распределением структурных элементов. Она состоит в нахождении полюсов конфигурационно усредненной резольвенты соответствующего гамильтониана [15]. Расчет указанной резольвенты может быть выполнен лишь в рамках некоторого приближения, определяемого спецификой исследуемой системы. Распространенным методом расчета квазичастичных состояний в неупорядоченных средах является приближение виртуального кристалла (ПВК) [15], которое позволяет выявлять 
особенности и трансформацию спектров элементарных возбуждений, обусловленную изменением концентрации дефектов в несовершенных структурах. В приближении ПВК усредненная резольвента равна резольвенте усредненного гамильтониана. В дальнейшем используем именно это приближение для расчета и анализа спектра экситонных возбуждений и особенностей зависимости химического потенциала от концентрации структурных дефектов исследуемой неидеальной решетки.

\section{Теоретическая модель}

Рассмотрим подробнее случай конденсации экситонов малого радиуса, экситонов Френкеля, которые возникают, например, в молекулярных кристаллах. Соответствующий математический аппарат описания данных возбуждений представлен в работе [16]. В ней показано, каким образом следует переходить от операторов рождения и уничтожения, определяющих возбуждения в изолированных молекулах, к экситонным операторам. Экситоны Френкеля подчиняются статистике Паули, однако при относительно малых плотностях эти квазичастицы можно считать бозонами, и, следовательно, возможно достижение экситонного конденсата [16].

Рассмотрим неидеальный топологически упорядоченный молекулярный кристалл с примитивной решеткой в модели [16] неподвижных молекул. Для расчета перенормированного спектра экситонного конденсата с взаимодействием между экситонами в гамильтониане электронной подсистемы данной структуры в узельном представлении в одноуровневой модели и приближении Гайтлера-Лондона следует оставить лишь следующие слагаемые $[17,18]$ :

$$
H=H_{2}+W_{3}+W_{4},
$$

где

$$
\begin{gathered}
H_{2}=\sum_{n} E_{n} B_{n}^{+} B_{n}=\frac{1}{2} \sum_{n, m}\left\langle 0 f\left|W_{n, m}\right| f 0\right\rangle B_{n}^{+} B_{m} \\
\equiv \sum_{n} E_{n} B_{n}^{+} B_{n}+\sum_{n, m} Q_{2 n m} B_{n}^{+} B_{m} \\
W_{3}=\sum_{n, m}\left\langle f 0\left|W_{n, m}\right| f f\right\rangle B_{n}^{+} B_{n} B_{m} \equiv \sum_{n, m} Q_{3 n m} B_{n}^{+} B_{n} B_{m}, \\
W_{4}=\sum_{n, m}\left\langle f f\left|W_{n, m}\right| f f\right\rangle B_{n}^{+} B_{m}^{+} B_{n} B_{m} \\
\equiv \sum_{n, m} Q_{4 n m} B_{n}^{+} B_{m}^{+} B_{n} B_{m}
\end{gathered}
$$

$f$ - номер возбужденного уровня, на котором формируется БЭК, $n$ и $m$ - номера узлов, $B_{n}^{+}, B_{n}-$ бозеоператоры рождения и уничтожения $f$-го возбуждения на узле $n, W_{n, m}$ - оператор кулоновского взаимодействия между молекулами, находящимися в узлах $n$ и $m$ соответственно, $E_{n}$ - энергия возбуждения молекулы, находящейся в $n-$-м узле. Поскольку экситоны Френкеля подчиняются статистике Паули и лишь приближенно являются бозе-частицами, то в выражении (1) должны присутствовать четырёхчастичные слагаемые, обусловленные кинематическим взаимодействием и описывающие взаимодействие конденсата с надконденсатными квазичастицами [16]. В настоящей работе рассматривается случай $T=0$, надконденсатные экситоны отсутствуют, и, следовательно, соответствующими слагаемыми можно пренебречь.

Матричные элементы оператора кулоновского взаимодействия $[17,18]$ имеют вид

$$
\begin{aligned}
Q_{2 n m} & \sim\left\langle f 0\left|W_{n m}\right| 0 f\right\rangle \sim\left\langle f\left|P_{n}\right| 0\right\rangle\left\langle 0\left|P_{m}\right| f\right\rangle, \\
Q_{3 n m} & \sim\left\langle f 0\left|W_{n m}\right| f f\right\rangle \sim\left\langle f\left|P_{n}\right| f\right\rangle\left\langle 0\left|P_{m}\right| f\right\rangle, \\
Q_{4 n m} & \sim\left\langle f f\left|W_{n m}\right| f f\right\rangle \sim\left\langle f\left|P_{n}\right| f\right\rangle\left\langle f\left|P_{m}\right| f\right\rangle,
\end{aligned}
$$

где $P_{n}$ - оператор дипольного момента молекулы $n$-го узла. Если учитывать взаимодействие только между конденсатными экситонами, то матричный элемент $\langle 0|P| f\rangle$, входящий в $Q_{2 n m}(5)$ и $Q_{3 n m}(6)$, будет отличен от нуля только для дипольно разрешенных состояний (светлые экситоны). Дополнительное ограничение накладывает множитель $\langle f|P| f\rangle$ в правой части (6) и (7). Он отличен от нуля только в кристаллах, у которых куб векторного представления содержит полносимметричное представление или, что то же самое, квадрат векторного представления содержит векторное представление. Примерами могут служить кристаллы с точечными группами симметрии $C_{3 V}, C_{4 V}, T$ и т.д. Ясно, что в кристаллах с центром инверсии этот матричный элемент будет равен нулю. Следовательно, в таких кристаллах $W_{3}=W_{4}=0$, и экситоны БЭК являются невзаимодействующими. Если изучается БЭК дипольно запрещенных (темных) экситонов, то матричный элемент $\langle 0|P| f\rangle=0$, а слагаемое четвертого порядка существенно только для состояний, квадрат неприводимых представлений которых содержит векторное представление. Такая ситуация возможна только в низкосимметричных кристаллах, у которых одна из компонент вектора преобразуется по полносимметричному представлению. В высокосимметричных кристаллах темные экситоны БЭК являются невзаимодействующими.

В дальнейшем для описания неидеальной структуры используем приближение ПВК. Таким образом, гамильтониан исследуемой системы $H$ следует заменить на конфигурационно усредненный гамильтониан $\bar{H}$. Полагая также, что данный кристалл является двухкомпонентным (т.е. исследуемая структура варьирует по составу), величины $E_{n}, Q_{2 n m}, Q_{3 n m}, Q_{4 n m}$ в (1) необходимо 
заменить следующими выражениями [15]:

$$
\begin{aligned}
E_{n} \rightarrow \bar{E}_{n} & =\sum_{\nu=1}^{2} c_{\nu} E^{(\nu)}, Q_{2(3,4) n m} \rightarrow \bar{Q}_{2(3,4) n m} \\
& =\sum_{\nu=1}^{2} \sum_{\lambda=1}^{2} c_{\nu} Q_{2(3,4) n m}^{(v, \lambda)} c_{\lambda}, \sum_{\nu=1}^{2} c_{\nu}=1,
\end{aligned}
$$

где $c_{v}$ - концентрация молекул $v$-го сорта, $E^{(v)}-$ энергия возбуждения молекулы данного сорта, $Q_{2(3,4) n m}^{(v, \lambda)}$ величины, соответствующие энергии взаимодействия молекулы $v$-го сорта в узле $n$ с молекулой $\lambda$-го сорта в узле $m$. В отличие от идеальной исследуемая структура имеет разупорядочение по составу, и поэтому гамильтониан (1) не является трансляционно инвариантным. Одна из методик получения спектра квазичастичных возбуждений неидеальной системы со случайным распределением структурных элементов состоит в нахождении полюсов конфигурационно усредненной резольвенты соответствующего гамильтониана [16]. Последняя является трансляционно инвариантной, и, следовательно, соответствующий спектр элементарных возбуждений можно характеризовать волновым вектором $\mathbf{k}$, а соответствующий гамильтониан имеет вид

$$
\bar{H}=\bar{H}_{2}+\bar{W}_{3}+\bar{W}_{4}=\sum_{\mathbf{k}} H(\mathbf{k})
$$

Слагаемое $\bar{H}_{2}$ диагонализуется путем преобразования Боголюбова-Тябликова. В итоге получаем экситонный гамильтониан в $k$-пространстве, в котором происходит конденсация квазичастиц в состояние $k=0$. Поскольку состояние БЭК экситонов при $\mathbf{k}=0$ является макроскопически заполненным, то в гамильтониане можно оставить только взаимодействие между конденсатными квазичастицами. В итоге получаем

$$
\begin{aligned}
& H(\mathbf{k}=0)=E_{e x}\left(c_{1}, c_{2}, \mathbf{k}=0\right) B^{+}(\mathbf{k}=0) B(\mathbf{k}=0) \\
& +\frac{1}{\sqrt{v}} Q_{3}\left(c_{1}, c_{2}, \mathbf{k}=0\right) B^{+}(\mathbf{k}=0) B(\mathbf{k}=0) B(\mathbf{k}=0) \\
& +\frac{1}{v} Q_{4}\left(c_{1}, c_{2}, \mathbf{k}=0\right) B^{+}(\mathbf{k}=0) B^{+}(\mathbf{k}=0) \\
& \times B(\mathbf{k}=0) B(\mathbf{k}=0),
\end{aligned}
$$

где

$$
\begin{aligned}
& E_{e x}\left(c_{1}, c_{2}, \mathbf{k}=0\right) \bar{E}_{\bar{n}}\left(c_{1}, c_{2}\right)+2 Q_{2}\left(c_{1}, c_{2}, \mathbf{k}=0\right), \\
& Q_{2(3,4)}\left(c_{1}, c_{2}, \mathbf{k}=0\right) \\
& =\left[\sum_{\bar{n}} \bar{Q}_{2(e 3,4) n m}\left(c_{1}, c_{2}\right) \exp \left(-i \mathbf{k} \cdot \mathbf{r}_{n m}\right)\right]_{\mathbf{k}=0}, \\
& B(\mathbf{k}=0)=\left[\frac{1}{\sqrt{N}} \sum_{n} B_{n} \exp \left(-i \mathbf{k} \cdot \mathbf{r}_{n}\right)\right]_{\mathbf{k}=0},
\end{aligned}
$$

Рис. 1. Графическое представление уравнения Дайсона для вычисления спектра при учете трехчастичного взаимодействия. Сплошная линия - ФГ конденсатного экситона при отсутствии взаимодействия, жирная сплошная - с учетом трехчастичного взаимодействия.

$N$ - число элементарных ячеек в объеме $V, \mathbf{r}_{n m}=$ $=\mathbf{r}_{n}-\mathbf{r}_{m}, \mathbf{r}_{n}, \mathbf{r}_{m}-$ векторы решетки, $E_{\text {ex }}-$ энергия экситона молекулярного кристалла в приближении ПВК; $Q_{2}\left(c_{1}, c_{2}, \mathbf{k}=0\right)$ - фурье-образ матрицы резонансного межмолекулярного взаимодействия, $Q_{3(4)}\left(c_{1}, c_{2}, \mathbf{k}=0\right)$ - фурье-образы матриц, описывающих 3(4)-частичные взаимодействия между конденсатными экситонами, $B^{+}(\mathbf{k}=0), B(\mathbf{k}=0)$ - операторы рождения и уничтожения конденсатных экситонов.

Для нахождения перенормированного спектра системы с $\mathbf{k}=0$ вычислим запаздывающую $M$-частичную функцию Грина (ФГ) (M - число конденсатных экситонов)с учетом слагаемых гамильтониана (8) третьего и четвертого порядков по экситонным операторам [19]. При этом слагаемые третьего порядка оказалось возможным учесть в лестничном приближении, как показано на рис. 1. Полученный спектр войдет в $M$-частичную $\Phi Г$ с множителем $M^{2}$. Метод учета слагаемого четвертого порядка представлен в работе [20].

Проделывая соответствующие вычисления по схеме $[21,22]$, для перенормированной энергии $M$-частиц конденсата получим двухуровневую систему:

$$
\begin{aligned}
E_{M}^{1}\left(c_{1}, c_{2}, \mathbf{k}\right. & =0)=M E_{e x}\left(c_{1}, c_{2}, \mathbf{k}=0\right) \\
& -\frac{M^{2} Q_{3}^{2}\left(c_{1}, c_{2}, \mathbf{k}=0\right)}{V E_{e x}\left(c_{1}, c_{2}, \mathbf{k}=0\right)} \\
& +M(M-1) \frac{Q_{4}\left(c_{1}, c_{2}, \mathbf{k}=0\right)}{V}, \\
E_{M}^{2}\left(c_{1}, c_{2}, \mathbf{k}\right. & =0)=2 M E_{e x}\left(c_{1}, c_{2}, \mathbf{k}=0\right) \\
& +\frac{M^{2} Q_{3}^{2}\left(c_{1}, c_{2}, \mathbf{k}=0\right)}{V E_{e x}\left(c_{1}, c_{2}, \mathbf{k}=0\right)} \\
& +M(M-1) \frac{Q_{4}\left(c_{1}, c_{2}, \mathbf{k}=0\right)}{V} .
\end{aligned}
$$

Таким образом, из соотношений $(11),(12)$ следует, что химический потенциал исследуемой неидеальной структуры имеет вид

$$
\begin{aligned}
\mu_{1} & =E_{M+1}^{(1)}-E_{M}^{(2)}=E_{e x}\left(c_{1}, c_{2}, \mathbf{k}=0\right) \\
& -\frac{Q_{3}^{2}\left(c_{1}, c_{2}, \mathbf{k}=0\right)}{E_{e x}\left(c_{1}, c_{2}, \mathbf{k}=0\right)}\left(2 n+\frac{1}{V}\right) \\
& +2 Q_{4}\left(c_{1}, c_{2}, \mathbf{k}=0\right) n
\end{aligned}
$$


(для нижайшего уровня) и

$$
\begin{aligned}
\mu_{2} & =E_{M+1}^{(2)}-E_{M}^{(2)}=2 E_{e x}\left(c_{1}, c_{2}, \mathbf{k}=0\right) \\
& +\frac{Q_{3}^{2}\left(c_{1}, c_{2}, \mathbf{k}=0\right)}{E_{e x}\left(c_{1}, c_{2}, \mathbf{k}=0\right)}\left(2 n+\frac{1}{V}\right) \\
& +2 Q_{4}\left(c_{1}, c_{2}, \mathbf{k}=0\right) n
\end{aligned}
$$

(для верхнего уровня). Здесь $n=\frac{M}{V}-$ плотность частиц в конденсате, $V$ - объем исследуемой системы. Из (12) и (14) следует, что в случае светлых экситонов слагаемые гамильтониана третьего порядка энергию квазичастиц на нижнем уровне системы понижают, а четвертого повышают, т.е. они ответственны за притяжение и отталкивание квазичастиц. Если вклад первых больше, то образование конденсата невозможно и образуются экситонные капли. В случае тёмных экситонов слагаемые третьего порядка вклада в энергию не дают, и образование конденсата возможно. При наличии слагаемых третьего порядка возможно появление конденсата светлых экситонов на верхнем энергетическом уровне, поскольку эти слагаемые ответственны за отталкивание. Статистические условия возникновения БЭК бозе-частиц рассматривались во многих работах (см., например, [7]).

Необходимо отметить, что выводы, сделанные в данном разделе, применимы и для двумерной подсистемы, а также для упомянутых выше систем с порами и квантовыми точками.

\section{Результаты и их обсуждение}

Детально исследуем зависимость химического потенциала от концентрации компонент, а также от разности их характеристик. Рассмотрение выполним для конкретного случая двухкомпонентной неидеальной системы с концентрациями структурных элементов соответственно $c_{1}$ и $c_{2}$. Таким образом, подставляя выражения (8) и $(10)$ в (12) с учетом $V \rightarrow \infty$, получим следующее соотношение для химического потенциала:

$$
\begin{aligned}
\mu_{1}\left(c_{1}, c_{2}\right) & =\sum_{i=1}^{2} c_{i}\left(E^{(i)}+2 \sum_{j=1}^{2} Q_{2}^{i j} c_{j}\right) \\
& -\frac{2\left(\sum_{i, j=1}^{2} c_{i} Q_{3}^{i j} c_{j}\right)^{2}}{\sum_{i=1}^{2} c_{i}\left(E^{(i)}+2 \sum_{j=1}^{2} Q_{2}^{i j} c_{j}\right)} n \\
& +2 \sum_{i, j=1}^{2} c_{i} Q_{4} c_{j} n,
\end{aligned}
$$

где

$$
Q_{2(3,4)}^{i j}=\left[\sum_{n} Q_{2(3,4) n m}^{i j} \exp \left(-i \mathbf{k} \cdot \mathbf{r}_{n m}\right)\right]_{\mathbf{k}=0}
$$

Условием образования конденсата является наличие отталкивающего взаимодействия между квазичастицами. Характер экситон-экситонного взаимодействия отражают второе и третье слагаемые в (15). При этом БЭК экситонов возможен лишь в случае, когда их сумма положительна. В случае темных экситонов в кристаллах без центра инверсии второе слагаемое отсутствует, а поскольку третье всегда положительное, то БЭК оказывается возможным. Появление БЭК в случае светлых экситонов обусловливается „конкуренцией“ в второго и третьего слагаемых в (15). Зависимости этих слагаемых от концентраций $c_{1}$ и $c_{2}=1-c_{1}$ параболические, однако характеристики парабол различны. Поэтому возможны ситуации, при которых для определенных значений параметров и концентраций знак суммарного взаимодействия может меняться на противоположный. В силу этого возможны различные условия образования БЭК.

Численное моделирование проведем для тех же значений параметров исследуемой структуры, что и в работах [21,22]:

$$
\begin{gathered}
E^{(1)}=4.5 \cdot 10^{4} \mathrm{~cm}^{-1} ; E^{(2)}=4 \cdot 10^{4} \mathrm{~cm}^{-1} \\
Q_{2}^{11}=3 \cdot 10^{4} \mathrm{~cm}^{-1} ; \\
Q_{2}^{22}=3.1 \cdot 10^{4} \mathrm{~cm}^{-1} ; \\
Q_{2}^{12} \approx Q_{2}^{12}=3.5 \cdot 10^{4} \mathrm{~cm}^{-1} ; \\
Q_{3}^{11}=2 \cdot 10^{4} \mathrm{~cm}^{-1} ; \\
Q_{3}^{22}=2.5 \cdot 10^{4} \mathrm{~cm}^{-1} ; \\
Q_{3}^{12} \approx Q_{3}^{21}=2.1 \cdot 10^{4} \mathrm{~cm}^{-1} ; \\
Q_{4}^{11}=0.4 \cdot 10^{4} \mathrm{~cm}^{-1} ; Q_{4}^{22}=0.3 \cdot 10^{4} \mathrm{~cm}^{-1} ; \\
Q_{4}^{12} \approx Q_{4}^{21}=0.1 \cdot 10^{4} \mathrm{~cm}^{-1} .
\end{gathered}
$$

На рис. 2 представлена ситуация (параметры модели указаны выше), когда при малых концентрациях взаимодействие экситонов отрицательное (притяжение), что приводит к образованию „капель“, а при больших положительное (отталкивание) и появляется возможность возникновения БЭК экситонов. При другом наборе параметров $[19,20]$ :

$$
\begin{gathered}
E^{(1)}=4.5 \cdot 10^{4} \mathrm{~cm}^{-1} ; E^{(2)}=4 \cdot 10^{4} \mathrm{~cm}^{-1} \\
Q_{2}^{11}=3.5 \cdot 10^{4} \mathrm{~cm}^{-1} ; Q_{2}^{22}=3.3 \cdot 10^{4} \mathrm{~cm}^{-1} \\
Q_{2}^{12} \approx Q_{2}^{12}=3 \cdot 10^{4} \mathrm{~cm}^{-1} \\
Q_{3}^{11}=2.7 \cdot 10^{4} \mathrm{~cm}^{-1} ; Q_{3}^{22}=2.3 \cdot 10^{4} \mathrm{~cm}^{-1} \\
Q_{3}^{12} \approx Q_{3}^{21}=1 \cdot 10^{4} \mathrm{~cm}^{-1} \\
Q_{4}^{11}=0.4 \cdot 10^{4} \mathrm{~cm}^{-1} ; Q_{4}^{22}=0.3 \cdot 10^{4} \mathrm{~cm}^{-1} \\
Q_{4}^{12} \approx Q_{4}^{21}=0.1 \cdot 10^{4} \mathrm{~cm}^{-1}
\end{gathered}
$$

как показано на рис. 3, имеет место образование БЭК экситонов для промежуточных значений концентрации 

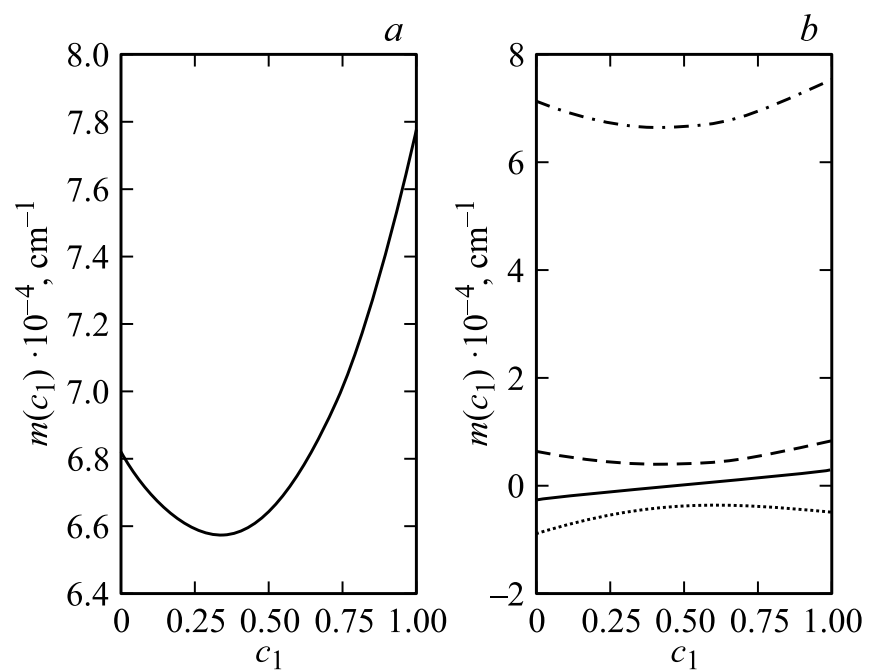

Рис. 2. $a-$ зависимость химического потенциала $\mu\left(c_{1}\right) \cdot 10^{4} \mathrm{~cm}^{-1}$ от концентрации $c_{1}\left(c_{2}=1-c_{1}\right)$ структурных компонент, $b$ - зависимости от концентрации первого (штрихпунктир), второго (пунктир) и третьего (штриховая) слагаемых в (15); сплошная линия - сумма второго и третьего слагаемых (15).
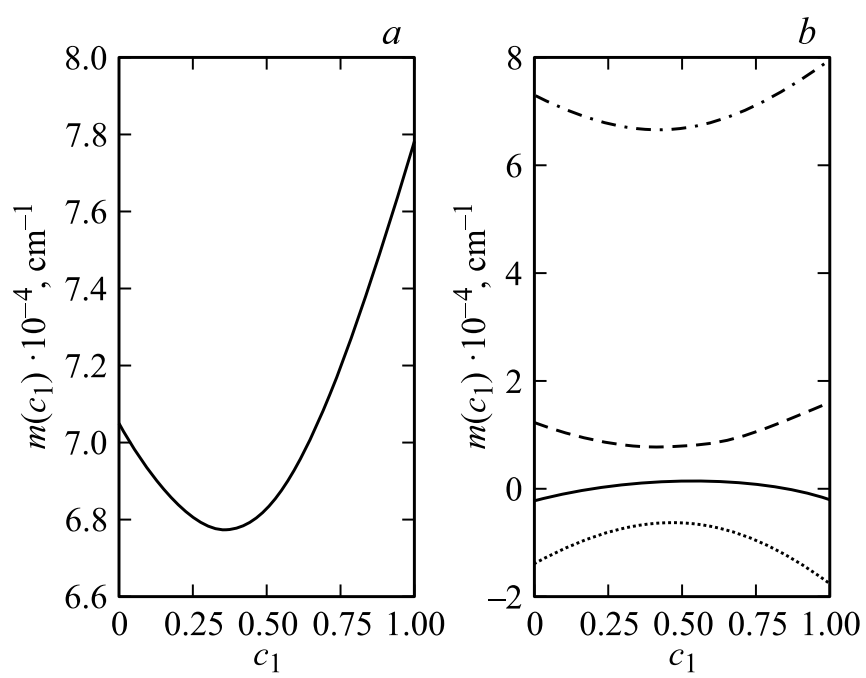

Рис. 3. $a-$ зависимость химического потенциала $\mu\left(c_{1}\right) \cdot 10^{4} \mathrm{~cm}^{-1}$ от концентрации $c_{1}\left(c_{2}=1-c_{1}\right)$ структурных компонент, $b$ - зависимости от концентрации первого (штрихпунктир), второго (пунктир) и третьего (штриховая) слагаемых в (15); сплошная линия - сумма второго и третьего слагаемых (15).

структурных дефектов. Причем при предельных значениях концентрации имеет место притяжение, и возникновение БЭК экситонов невозможно. Следует отметить, что если распределение компонент в кристалле неоднородно, то в обоих случаях возможно появление хаотических пространственно разделенных областей с БЭК экситонами (БЭК экситонных „капель“), между которыми находятся запрещенные области. Отметим, что этот вывод справедлив при произвольных значениях концентраций $n$, поскольку оба слагаемые имеют одинаковую зависимость от плотности.

Если в кристалле слагаемое третьего порядка доминирует, то БЭК светлых экситонов на нижнем уровне невозможен при любых концентрациях составляющих элементов. В то же время возможен БЭК на верхнем уровне, однако существенным фактором становится время жизни квазичастиц. Темные экситоны могут конденсироваться, но поскольку они не могут люминесцировать, то их наблюдение затруднено. Для обнаружения таких состояний необходимо применять другие методики, в частности рассматривать нелинейные оптические процессы с участием БЭК $[8,9]$.

\section{Заключение}

В работе исследована возможность появления экситонного БЭК в модельной неидеальной решетке молекулярного кристалла. Проведен соответствующий анализ спектра экситонных возбуждений. В рамках ПВК выполнено численное моделирование зависимости химического потенциала от концентрации структурных дефектов и на этой основе изучены особенности появления экситонного конденсата в неидеальной системе. Показано, при каких условиях возможно появление БЭК светлых и темных экситонов. Полученные результаты представляют интерес при интерпретации экспериментальных данных и получении соответствующих оптических характеристик.

\section{Финансирование работы}

Исследования выполнены в рамках госбюджетно финансируемого проекта Донецкого физико-технического института им. А.А. Галкина „Формирование структуры и свойств перспективных многофункциональных материалов".

\section{Конфликт интересов}

Авторы заявляют, что у них нет конфликта интересов.

\section{Список литературы}

[1] Москаленко C.A. Бозе-эйнштейновская конденсация экситонов. Кишинев: Штиинца, 1970. 168 с.

[2] Moskalenko S.A., Snoke D.W. Bose-Einstein Condensation of Excitons and Biexcitons and Coherent Nonlinear Optics with Excitons. Cambridge: Cambridge University Press, 2000.

[3] Акопян И.Х., Гросс Е.Ф., Разбирин Б.С. // Письма в ЖЭТФ. 1970. Т. 12. С. 366-372.

[4] Kato Y., Goto T., Fuji T., Ueta M. // J. Phys. Soc. Japan.1974. V. 36. N 1. P. $169-176$.

[5] Peyghambarian N., Chase L.L. // Phys. Rev. B. 1983. V. 27. N 4. P. 2325-2345.

[6] KogarAnshul, Rak Melinda S., Vig Sean etal. // Science. 2017. V. 358. P. 1314-1317. 
[7] Combescot Monique, Combescot Roland, Dubin François // Rep. Prog. Phys. 2017. V. 80. P. 066501.

[8] Rumyantsev V.V., Zavorotnev Yu.D., Popova O.Yu. // J. Photonic Materials and Technology. 2018. V. 4. P. 39-48.

[9] Заворотнев Ю.Д., Овандер Л.Н. // Известия РАН. Серия физическая. 2005. Т. 69. С. 984-986.

[10] Сотников А.Г., Середа К.В., Слюсаренко Ю.В. // Физика низких температур. 2017. Т. 43. С. 172-181.

[11] Rumyantsev V.V., Fedorov S.A., Gumennyk K.V., Sychanova M.V., Kavokin A.V. // Nature. Sci. Rep. 2014. V. 4. P. 6945.

[12] Румянщев В.В., Федоров С.А., Гуменник К.В. // ФТТ. 2017. T. 59. C. 741-747.

[13] Rumyantsev V.V., Fedorov S.A., Gumenny k K.V., Gurov D.A., Kavokin A.V. //Superlattices and Microstructures. 2018. V. 120. P. 642-649.

[14] Rumyantsev V.V., Fedorov S.A., Gumennyk K.V., Paladyan Yu.A. // Physica B: Condensed 4 Matter. 2019. V. 571. N 15. P. 296-300.

[15] Займан Джс. Модели беспорядка. М.: Мир, 1982.

[16] Агранович В.М. Теория экситонов. М.: Наука, 1968. 328 с.

[17] Агранович В.М., Галанин М.Д. Перенос энергии электронного возбуждения в конденсированных средах. М.: Наука, 1978. $383 \mathrm{c}$.

[18] Заворотнев Ю.Д., Овандер Л.Н. Нелинейные оптические эффекты в молекулярных кристаллах. Донецк: НордПресс, 2005. 274 c.

[19] Абрикосов А.А., Горьков Л.П., Дзялошинский И.Е. Методы квантовой теории поля в статистической физике. М.: ГИФМЛ, 1962. $443 \mathrm{c.}$

[20] Zavorotnev Yu.D., Popova O.Yu. // J. Photonic Materials and Technology. 2015. V. 1. P. 10-14.

[21] Овандер Л.Н. // УФН.1965. Т. 86. С. 3-39.

[22] Zavorotnev Yu.D., Ovander L.N. // Phys. Stat. Sol. 1975. V. 68. P. 443-452. 